\title{
Efecto del peso al nacer, tamaño de camada y posición en la ubre sobre el crecimiento de cerdos durante la lactancia y engorda
}

\section{Effect of birth weight, litter size and nipple position on growth of pigs during lactation and fattening}

\author{
J uan Uriel Rendón del Águilaa, Roberto Gustavo Martínez-Gambaa*, Marco Antonio Herradora Lozanoa, \\ María Alonso-Spilsbury ${ }^{b}$
}

\begin{abstract}
RESUMEN
Este estudio se realizó para conocer el crecimiento en lactancia y engorda de lechones nacidos con bajo peso, en 32 camadas de alta prolificidad, para lo cual se establecieron tres categorías de peso al nacer PBAJO, PMEDIO y PALTO y tres de tamaño de camada BAJ A, MEDI A y ALTA; se identificó el pezón asegurado por el lechón durante el nacimiento y la lactancia, y se determinaron los efectos de dichas categorías en variables de peso y ganancia de peso durante la lactancia y la etapa de crecimiento; así como en la grasa dorsal, la profundidad del lomo y el rendimiento en canal a los 160 días. Las camadas de prolificidad ALTA tuvieron una mayor proporción de lechones de bajo peso ( $26 \%$ ) contra $4 \%$ en camadas de BAJ A prolificidad; los lechones de PBAJ O demoraron más en succionar los pezones (50.37 min) que los de PMEDIO y PALTO (32.44 y 24.34 min respectivamente) y el $67 \%$ de ellos se posicionaron en mayor proporción en los pezones posteriores de la cerda. Los lechones de PBAJ O tuvieron menor ganancia de peso en lactancia, 181 g/ día contra 211 y 240 g/ día en categorías de PMEDIO y PALTO respectivamente, y menor peso a 160 días de edad ( $86.6 \mathrm{~kg}$ vs 95.7 y $101.1 \mathrm{~kg}$ ). Se concluye que en camadas de alta prolificidad, los lechones de bajo peso tienen el desempeño productivo más bajo independientemente del tamaño de su camada al nacer y el pezón en el que lactaron.
\end{abstract}

PALABRAS CLAVE: Bajo peso al nacer, Lechones, Pezón, Tamaño de camada.

\begin{abstract}
Growth during lactation and feeding was evaluated for low birth weight (runt) piglets in 32 high prolificacy litters using three birth weight categories (low, medium, and high) and three litter sizes (small, medium and large). The teat secured during birth and lactation by each studied piglet was identified. An analysis was done of the effects of the birth weight and litter size categories the variables weight and weight gain during lactation and feeding; back fat; loin depth; and carcass yield at $160 \mathrm{~d}$. High prolificacy litters had a higher proportion of low birth weight piglets $(26 \%)$ than low prolificacy litters (4\%). Low birth weight piglets took longer to suck the teat $(50.37 \mathrm{~min})$ than the medium $(32.44 \mathrm{~min})$ and high weight piglets (24.34 min). Most (67 \% ) of the runts were positioned largely on the sow's posterior teats. Low weight piglets exhibited lower weight gain (181 $\mathrm{g} / \mathrm{d}) \mathrm{than}$ the medium $(211 \mathrm{~g} / \mathrm{d})$ and high weight piglets $(240 \mathrm{~g} / \mathrm{d})$. Their weight at $160 \mathrm{~d}(86.6 \mathrm{~kg})$ was also lower than in the medium $(95.7 \mathrm{~kg})$ and high weight piglets $(101.1 \mathrm{~kg})$. In high prolificacy litters, low weight piglets (runts) exhibited the lowest growth performance, independent of litter size and lactation teat.
\end{abstract}

KEY WORDS: Low birth weight, Piglets, Teat, Litter size.

Recibido el 18 de febrero de 2015. Aceptado el 5 de agosto de 2015.

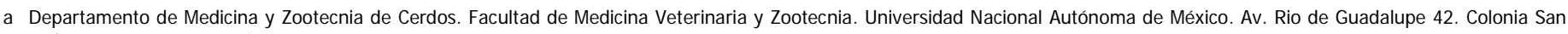
Juan de Aragón. 07950. México D.F.

b Departamento de Producción Agrícola y Animal. Universidad Autónoma Metropolitana, Unidad Xochimilco. México.

* Autor de correspondencia: rmgamba@yahoo.com.mx. 
El incremento del tamaño de camada resulta desfavorable para la sobrevivencia y la vitalidad del lechón ${ }^{(1)}$, por lo que tiene un impacto negativo en la mortalidad pre-destete ${ }^{(2)}$, el peso al nacimiento y la uniformidad en la camada, aspecto de suma importancia en la sobrevivencia del lechón ${ }^{(3,4)}$. Es claro que el incremento en el tamaño de la camada, disminuye la media de peso al nacer y aumenta la proporción de lechones de bajo peso al nacimiento(5).

Por lo anterior, es claro que la sobrevivencia del lechón, es de gran importancia en líneas de cerdas hiper-prolíficas, donde la variación de peso entre los miembros de sus camadas, es una determinante de la mortalidad y la ganancia diaria pre-destete, y con diferencias de peso y características de la canal en etapas posteriores ${ }^{(6)}$. El peso al nacer y su dispersión, son aspectos que cada vez tienen mayor relevancia en la producción porcina, y la estrategia de introducir cerdas hiper-prolíficas como un medio de incrementar el número de nacidos, requiere de una evaluación crítica en el contexto de la eficiencia de toda la cadena de producción porcina.

Es importante conocer las características del desarrollo de los lechones de bajo peso, poder minimizar sus efectos, y buscar estrategias económicamente viables para lograr que sean funcionales productiva y financieramente. Por lo tanto, el objetivo de este estudio fue evaluar el desempeño de los lechones al nacer, según una categorización de peso y evaluar su desarrollo en etapas subsecuentes.

El trabajo se realizó en una unidad comercial de producción porcina con 1,700 hembras de uno y dos partos, localizada en el estado de Veracruz a $18^{\circ} 47^{\prime}$ $\mathrm{N}$ y $96^{\circ} 34^{\prime} \mathrm{O}$, con clima Am(f)(i')gw.

Se escogieron al azar antes del parto las camadas de 32 cerdas F1 (50\% Landrace X 50\% Large White) nacidas durante dos semanas, 16 cerdas de primer parto y 16 de segundo. Los lechones evaluados desde el nacimiento fueron producto de inseminaciones con semen proveniente de machos de una línea Pietrain.

Para el análisis de la información, se tomaron los datos de las 32 camadas. Se registró el número de camada, la identificación de la cerda, fecha del parto, número de parto de la cerda y duración del parto cuando el último lechón de cada cerda fue expulsado, así como lechones nacidos totales por camada (LNT).

Una vez terminado cada parto, se tatuó toda la camada con los mismos números consecutivos que se les marcaron al nacer en la oreja derecha; se registró el número correspondiente a cada lechón, se registró el sexo y se pesó en una balanza electrónica modelo (OCS-L, Crane Scale) registrando el peso al nacer (PN); una vez identificados y pesados se volvieron a ubicar de inmediato en la parte posterior de la cerda para no interferir con su búsqueda de un pezón.

Recabados todos los datos de parto, se calculó la media y la desviación estándar (DE) de PN; con esta información se procedió a restar y a sumar a la media una DE para generar los tres categorías de PN, tratando así de obtener una categoría de lechones clasificados como de bajo peso (PBAJO) y los que estuvieron por arriba se clasificaron como de peso alto (PALTO). Los restantes se clasificaron como de peso medio (PMEDIO). Esta categorización se mantuvo durante toda la prueba.

Con los datos del número de LNT, se hizo una categorización según la prolificidad de las camadas, que debido a las características de la línea genética y a información analizada previamente, se estableció como de BAJA prolificidad a los partos con 12 o menos, MEDI A a las camadas entre 13 y 15 , y ALTA 16 o más lechones.

Se registró la hora en que cada lechón logró asegurar algún pezón ${ }^{(7)}$; se determinó el número de pezón succionado, para lo cual se numeraron del 0 al 9 los pezones de la ubre derecha y del 10 al 19 para la izquierda. Para determinar el tiempo de latencia o letargo, se hizo una resta usando el registro de la hora de aseguramiento menos el registro de la hora de nacimiento. Una vez terminada esta etapa los lechones recibieron el manejo convencional en la granja. Durante la lactancia se realizó el registro de las bajas, la edad y el peso del animal al momento que se encontró muerto, basándose en las características del animal ${ }^{(8)}$.

Previo al destete, para cada lechón se hizo el registro del pezón que succionaba; con esta 
información y de acuerdo a su categoría de peso, se hizo un análisis de qué posicionamiento tuvieron en las tetas de acuerdo a su categoría de peso considerando pezones anteriores (ANT) los numerados: 1, 2, 3, 10, 11 y 12; medios (MED), los pezones $4,5,6,13$, 14 y 15 y posteriores (POS), los números 7, 8, 9, 16, 17, 18 y 19.

El destete se realizó a los 21 días, momento en que se registró el peso (PD) y se obtuvo la ganancia media diaria de peso en lactancia (GMDL). Posteriormente se registró el peso de cada individuo y se obtuvo la ganancia media diaria a los 70,85 , 100, 115, 130, 145 y 160 días. Al día 160, se realizó la medición de grasa dorsal (GD) y profundidad (PL) de lomo en P2, con un ultrasonido (Preg Alert Pro, Renco). Con esta información, se realizó la estimación de rendimiento magro $(\mathrm{RM})^{(9)}$.

Se evaluaron las siguientes variables por categoría de peso y tamaño de camada: número de animales, promedio de peso individual al nacimiento (PN), tiempo de latencia (TL) para aseguramiento del pezón, porcentaje de fidelidad (F) del lechón en la ubre, mortalidad (Mo) en lactancia, peso al destete (PD) y ganancia media diaria en lactancia (GMDL); peso individual (PI) y ganancia diaria de peso (GDP) a los 70, 85, 100, 115, 130, 145 y 160 días, rendimiento de tejido magro estimado (RM), grasa dorsal (GD) y profundidad de lomo (PL).

Para PN se hizo un análisis de varianza (ANDEVA) según la categorías de peso individual al nacimiento, empleando como bloque el número de parto de la madre; para peso al destete se hizo un ANDEVA con un modelo completamente al azar empleando como covariable PN y como bloque el parto de la madre. El TL se analizó por medio de ANDEVA según la categoría de peso y empleando como bloque el número del parto de la madre. Se determinó el porcentaje de lechones de cada categoría de PN y su posicionamiento en la ubre, se hizo la trasformación de los porcentajes obteniendo la raíz cuadrada del arco seno y se realizó un análisis no paramétrico de Wilcoxon dentro de cada categoría de peso al nacer, y un análisis de Krushkall-Wallis para los porcentajes dentro del grupo de peso para el posicionamiento del pezón ${ }^{(10)}$.
De igual manera se realizó un ANDEVA para PD y GMDL por categoría de PN, empleando como covariable el peso al nacer. Además se realizó una correlación entre el número de $\mathrm{LNT}$ con GMDL ${ }^{(10)}$. Por último, para PI y GMD a 70, 85, 100, 115, 130, 145 y 160 días se realizó un análisis de varianza para medidas repetidas. Se realizó un análisis de varianza para las variables GD, PL y RM por categoría de peso. Para todos los análisis estadísticos se empleó el programa informático J MP versión 4.0.2(11).

Como resultados, se obtuvo el peso de 417 lechones, 216 de camadas de primer parto y 201 de segundo. Con respecto a LNT fueron $14.13 \pm 2.94$ para cerdas de primer parto, $13.79 \pm 2.93$ para las de segundo, y de $14.24 \pm 2.94$ para ambas, variando entre las hembras de primero y segundo parto $(P<0.0001)$. El peso promedio al nacimiento fue de $1.274 \pm 0.288 \mathrm{~kg}$. La categoría PBAJ O incluyó a 70 animales con menos de $0.986 \mathrm{~kg}$, la de PMEDIO a 287 animales de 0.986 a $1.562 \mathrm{~kg}$ y PALTO a 60 lechones con peso mayor a $1.562 \mathrm{~kg}$.

En la categorización de las camadas por su nivel de prolificidad, hubo 10 camadas para la categoría de BAJA prolificidad (con 12 o menos LNT) con 97 lechones al nacimiento de $1.46 \mathrm{~kg} \pm 0.295 \mathrm{~kg}$, con un valor mínimo de $0.58 \mathrm{~kg}$ y un valor máximo de $2.08 \mathrm{~kg}$. Para la categoría de MEDIA hubo 13 camadas con 176 lechones de $1.24 \mathrm{~kg} \pm 0.247$, con un valor mínimo de $0.64 \mathrm{~kg}$ y un valor máximo de $1.86 \mathrm{~kg}$, y para la categoría de ALTA prolificidad (16 o más LNT) hubo 9 camadas con 144 lechones de $1.19 \mathrm{~kg} \pm 0.278$, con valor mínimo de $0.38 \mathrm{~kg}$ y valor máximo de $1.86 \mathrm{~kg}$.

En camadas de prolificidad BAJA no hubo individuos de la categoría de PBAJ O, mientras que en las más numerosas no hubo de PALTO $(P<0.001)$. Algunos autores consideran de bajo peso aquéllos debajo de $1.14 \mathrm{~kg}^{(8)}$ y de $1.20 \mathrm{~kg}^{(12,13)}$ y otros excluyen como bajo peso a los lechones de menos $800 \mathrm{~g}^{(14)}$ o de $750 \mathrm{~g}^{(15)}$, sin embargo no mencionan su metodología para determinar el establecimiento de sus categorías.

Así mismo, se considera que el nivel de prolificidad de las madres tiene influencia sobre el peso promedio de los lechones y su variabilidad, pero solo unos autores relacionan la prolificidad con 
el peso de los lechones ${ }^{(15)}$. En el presente estudio, las cerdas de primer parto tuvieron mayor proporción de lechones de bajo peso, y las de segundo ciclo mayor impacto en la variabilidad del peso. Conforme las camadas incrementaron en prolificidad, el peso medio de los lechones disminuyó, lo que concuerda con autores ${ }^{(5,16)}$, que señalan entre 20 y $25 \%$ de lechones de bajo peso $(\leq 1.00 \mathrm{~kg}$ ) en camadas $\geq 15 \mathrm{LNV}$, lo que coincide con los porcentajes encontrados en este estudio dentro de la categoría de alta prolificidad ( $\geq 16$ LNT).

El TL se presenta en el Cuadro 1 donde se observa que los lechones de la categoría PBAJO tardaron más en asegurar el pezón independientemente del número de parto.

En el Cuadro 2 se observa que lechones de PBAJ O se posicionaron en una mayor proporción en los pezones ANT y MED, mientras que los de PMED y PALTO lo hicieron en los pezones de ubicación ANT. Con respecto al tiempo de latencia los animales de PBAJO, demoraron más tiempo en hacer su primer aseguramiento de pezón, lo que es

Cuadro 1. Tiempo promedio de aseguramiento de pezón según la categoría de peso y el número de parto

\begin{tabular}{llll}
\hline & \multicolumn{3}{c}{ Número de parto } \\
\cline { 2 - 4 } & \multicolumn{1}{c}{2} & Total \\
\cline { 2 - 4 } Categoría & & & \\
\hline de peso & & & $0: 50: 37^{\mathrm{a}}$ \\
Bajo & $0: 52: 56^{\mathrm{ax}}$ & $0: 47: 04^{\mathrm{ax}}$ & $0: 32: 44^{\mathrm{a}}$ \\
Medio & $0: 32: 37^{\mathrm{bx}}$ & $0: 32: 52^{\mathrm{bx}}$ & $0: 29: 34^{\mathrm{a}}$ \\
Alto & $0: 31: 34^{\mathrm{bx}}$ & $0: 28: 39 \mathrm{bx}$ & $0: 35: 03$ \\
Total & $0: 36: 06^{\mathrm{x}}$ & $0: 33: 54^{\mathrm{x}}$ &
\end{tabular}

Literales diferentes en cada columna $(a, b)$ y renglón $(x)$ indican diferencia $(P<0.01)$. concordante con la literatura ${ }^{(17,18)}$. Es importante mencionar que las categorías de PBAJO y PMEDIO, mostraron la mayor variabilidad en términos de desviación estándar en el tiempo empleado, lo que indica la variabilidad que hay en los animales de cada categoría con respecto a esta variable. En relación al posicionamiento del lechón en la ubre, el comportamiento de amamantamiento del recién nacido es desordenado y diferenciado del patrón organizado de los días posteriores al parto; los pares de tetas 6 y 7 son los más empleados en una primera succión, y los animales de buen peso se trasladan posteriormente a los pezones anteriores, desplazando a los de bajo peso a los pezones posteriores $^{(7)}$. En la presente prueba se encontró un comportamiento muy similar, los primeros pezones succionados eran los del área posterior, por ser los primeros encontrados. Sin embargo, si bien los lechones pesados se posicionaron en los anteriores, hubo animales de las tres categorías de peso, ubicados en las tres categorías de posición en la ubre; lo que indica que la capacidad de estimular y extraer calostro y leche es más importante que el posicionamiento del lechón en la ubre. En lo que respecta al porcentaje de fidelidad a la teta registrada al primer aseguramiento y de la teta asegurada al destete, el 94 \% cambió de teta y solo el $5.6 \%$ se mantuvieron con la misma.

Se registró la muerte de 18 lechones (4.6\%), 11 se clasificaron como inanición de 2.82 días y $0.847 \mathrm{~kg}$; y siete como aplastados con 5.14 días y $1.02 \mathrm{~kg}$. El $67 \%$ (12) de las muertes fue PBAJO y $33 \%$ (6) de PMEDIO. Lo anterior concuerda con reportes que indican que los lechones de bajo peso al nacimiento, tienen una alta probabilidad de mortalidad, debido al aplastamiento y la

Cuadro 2. Porcentaje de lechones según categoría de peso al nacimiento y posición del pezón en la ubre

\begin{tabular}{|c|c|c|c|c|c|c|c|c|}
\hline \multirow[b]{3}{*}{ Posición en la ubre } & \multicolumn{4}{|c|}{ Categoría de peso } & & & & \\
\hline & \multicolumn{2}{|c|}{ Bajo } & \multicolumn{2}{|c|}{ Medio } & \multicolumn{2}{|c|}{ Alto } & \multicolumn{2}{|c|}{ Total } \\
\hline & $\mathrm{n}$ & $\%$ & $\mathrm{~N}$ & $\%$ & $n$ & $\%$ & $\mathrm{n}$ & $\%$ \\
\hline Anterior & 16 & $39.02^{a}$ & 94 & $47.24^{a}$ & 22 & $50.00^{a}$ & 132 & $46.48^{a}$ \\
\hline Media & 19 & $46.34^{a}$ & 77 & $38.69^{a}$ & 17 & $38.64^{a}$ & 113 & $39.79^{a}$ \\
\hline Posterior & 6 & $14.63^{b}$ & 28 & $14.07^{b}$ & 5 & $11.36^{b}$ & 39 & $13.73^{b}$ \\
\hline Total & 41 & 100.00 & 199 & 100.00 & 44 & 100.00 & 284 & 100.00 \\
\hline
\end{tabular}

ab Literales diferentes en cada columna indican diferencia $(P<0.05)$.. 
inanición ${ }^{(3,17)}$. Sin embargo, es importante considerar que no necesariamente un lechón de categoría de bajo peso será una baja.

Al momento del destete se pesaron 370 animales, ya que el personal de la granja anticipó el destete de 17 animales de dos camadas. El promedio de días de destete fue de 21.61 días, no existiendo diferencia por categoría de peso y número de parto $(P>0.001)$. En el Cuadro 3 se presenta el promedio de PD y GMDL según la categoría de peso y el número de parto. En la prueba se aprecia cómo a mayor LNT la GMDL va disminuyendo $\left(R^{2}=0.102\right.$; $P<0.001)$ en camadas de ambos números de parto de la cerda ( $P>0.05)$.

Con respecto a PD según las categorías de peso y la posición del lechón durante la lactancia, resalta

Cuadro 3. Promedio de peso al destete (PD) y ganancia media diaria en lactancia (GMDL) de lechones en diferentes categorías de peso al nacer

\begin{tabular}{lrcccc}
\hline Categoría & \multicolumn{1}{c}{$\mathrm{n}$} & $\mathrm{PD}(\mathrm{kg})$ & $\mathrm{DE}$ & $\mathrm{GMDL}(\mathrm{kg})$ & $\mathrm{DE}$ \\
\hline Bajo & 49 & $4.92^{\mathrm{a}}$ & 1.093 & 0.181 & 0.006 \\
Medio & 272 & $5.96^{\mathrm{b}}$ & 1.224 & 0.211 & 0.002 \\
Alto & 49 & $6.93^{\mathrm{c}}$ & 1.335 & 0.240 & 0.006 \\
Total & 370 & 5.95 & 1.326 & 0.211 & 0.004 \\
\hline
\end{tabular}

DE = desviación estándar.

abc Literales diferentes en la misma columna indican diferencia $(P<0.01)$. que para PBAJO y PALTO la posición en la ubre no tuvo efecto en el peso al destete, no así para PMEDIO donde los lechones colocados en posición anterior tuvieron un menor peso ( $P<0.01$ ) (Cuadro 4).

En el Cuadro 5 se presenta la GMDL por categoría de peso y el posicionamiento en la ubre, donde se observa que este último no tiene efecto dentro de cada categoría de PN y que los animales de PALTO tuvieron mejor GMDL independientemente de la posición del pezón.

A medida que aumenta la escala de prolificidad disminuyen PD y GMD. En el análisis por sexo se encontró que los lechones de PBAJO tuvieron una menor GMDL independientemente del sexo $(P<0.001)$.

En este estudio los animales con mejor categoría de peso, presentaron una mejor GMDL, mientras que a mayor categoría de prolificidad, fue menor. Lo anterior se explica, ya que en las camadas numerosas hay una mayor ausencia de los lechones durante los eventos de succión, con una correlación positiva entre el tiempo de duración de la bajada de la leche y la GMD. Los intervalos de pre y postmasaje a la bajada de la leche, son más cortos cuando hay limitantes de espacio por lechón en la camada, lo que se puede extrapolar a camadas numerosas ${ }^{(18)}$.

Cuadro 4. Peso promedio individual al destete (PD) y desviación estándar, según la categoría de peso y posicionamiento en la ubre $(\mathrm{kg})$

\begin{tabular}{ccccccc}
\hline & \multicolumn{5}{c}{ Categorías de peso al nacer } \\
\cline { 2 - 7 } Posición en la ubre & $\mathrm{n}$ & $\mathrm{B}$ Pajo & $\mathrm{n}$ & $\mathrm{PD}$ & $\mathrm{n}$ & Alto \\
\cline { 2 - 7 } Anterior & 16 & $4.95 \pm 0.5277^{\mathrm{ax}}$ & 94 & $6.33 \pm 0.198^{\mathrm{ax}}$ & 22 & $7.25 \pm 0.288^{\text {ay }}$ \\
Media & 19 & $4.70 \pm 0.577^{\mathrm{ax}}$ & 77 & $6.34 \pm 0.212^{\text {ay }}$ & 17 & $6.71 \pm 0.430^{\text {ay }}$ \\
Posterior & 6 & $5.26 \pm 0.645^{\mathrm{ax}}$ & 28 & $5.76 \pm 0.345^{\mathrm{bx}}$ & 5 & $7.10 \pm 0.645^{\mathrm{ay}}$ \\
\hline
\end{tabular}

Literales diferentes en la misma columna $(a, b)$ y renglón $(x, y)$ indican diferencia $(P<0.01)$.

Cuadro 5. Ganancia media diaria de peso en lactancia (GMDL) en kilos, según la categoría de peso y el posicionamiento en la ubre

\begin{tabular}{ccccccc}
\hline & \multicolumn{5}{c}{ Categorías de peso al nacer } \\
\cline { 2 - 7 } & \multicolumn{7}{c}{ Bajo } & Medio & Alto \\
\cline { 2 - 7 } Posición en la ubre & $\mathrm{n}$ & $\mathrm{GMDL}$ & $\mathrm{n}$ & $\mathrm{GMDL}$ & $\mathrm{n}$ & $\mathrm{GMDL}$ \\
\hline Anterior & 16 & $0.225 \pm 0.029^{\mathrm{ax}}$ & 94 & $0.226 \pm 0.007$ ax & 22 & $0.243 \pm 0.011^{\text {ay }}$ \\
Media & 19 & $0.164 \pm 0.022^{\text {ax }}$ & 77 & $0.216 \pm 0.008^{\text {ay }}$ & 17 & $0.227 \pm 0.016^{\text {ay }}$ \\
Posterior & 6 & $0.167 \pm 0.025^{\text {ax }}$ & 28 & $0.201 \pm 0.013^{\text {bx }}$ & 5 & $0.249 \pm 0.052^{\text {ay }}$ \\
\hline
\end{tabular}

Literales diferentes por columna (a) y renglón $(x, y)$ indican diferencia $(P<0.01)$; en el caso del renglón Posterior $(P<0.05)$. 
En el Cuadro 6 se observa que los cerdos de PBAJ O tuvieron el menor peso en todos los pesajes. No se encontró interacción entre la categoría de peso y el sexo en ninguno de los pesajes ( $P>0.05)$, ni efecto del número de parto de la madre. Se encontraron diferencias en el peso por sexo a los $100,115,130$ y 160 días $(P<0.05)$. Con respecto a la GMD se encontraron diferencias a 100 y 115 días a favor de PALTO $(P<0.05)$. Para GD, PL y RM no se encontraron diferencias por categoría de peso ni tamaño de camada ( $P>0.05)$.

Estos resultados permiten visualizar las diferencias entre las categorías de peso al nacer, y concuerda con un estudio donde se reportan mediciones de peso a 28,70, 133 y 180 días de vida, y donde se puede apreciar que el comportamiento de las tres categorías es similar al del presente estudio ${ }^{(18)}$.

Con respecto al espesor de grasa dorsal, profundidad del lomo y rendimiento de tejido magro de acuerdo a su categoría de peso, lechones de bajo peso tienen desventajas productivas, pero además, hay reportes que mencionan que desde el nacimiento en estos animales hay factores prenatales que afectan el número total de fibras musculares, que tienen un efecto permanente en el crecimiento postnatal de los músculos de los cerdos $^{(13)}$. En el presente estudio no se encontraron diferencias entre los tres grupos en GD y PL; y en lo que respecta a $\mathrm{RM}$, los resultados fueron similares y concuerdan con otros trabajos ${ }^{(13,18)}$.
Sin embargo en cuanto a peso individual, en las categorías de PBAJO y PMEDIO hubo animales que alcanzaron a los de PALTO. Así mismo, en las categorías de PALTO y PMEDIO, hubo animales lentos en su desarrollo de crecimiento, que tuvieron pesos individuales similares a los de sus compañeros de categorías inferiores de peso. Lo que indica que el peso al nacimiento no es la única determinante del desempeño productivo, sino que los eventos de cada etapa, tanto en la cerda como en el lechón influyen en su desempeño final.

Se concluye que las cerdas de alta prolificidad tuvieron una mayor proporción de lechones de bajo peso, que demoraron más en ingerir calostro y se posicionaron en mayor proporción en los pezones posteriores. Los lechones de bajo peso tuvieron en promedio una menor ganancia de peso en lactancia y un menor peso a edad a sacrificio.

Este estudio mostró que el tener metas de alta prolificidad puede originar efectos complejos de contrarrestar en toda la cadena productiva, por lo que hay que ser precisos en el manejo de cerdas y lechones, para minimizar estos efectos.

\section{LITERATURA CITADA}

1. Douglas SL, Edwards SA, Sutcliffe E, Knap PW, Kyriazakis I. Identification of risk factors associated with poor lifetime growth performance in pigs. J Anim Sci 2013; 91:4123-4132.

2. Heim G, Mellagi APG, Bierhals T, De-Souza LP, De-Fries HCC, Piuco $P$, et al. Effects of cross-fostering within $24 \mathrm{~h}$ after birth on preweaning behaviour, growth performance and survival rate of biological and adopted piglets. Livest Sci 2012;150:121-127.

Cuadro 6. Promedio de peso en kilos (pp) y desviación estándar (DE) a los 75, 85, 100, 115, 130, 145 y 160 días de edad por categoría de peso al nacimiento

\begin{tabular}{|c|c|c|c|c|c|c|c|c|c|c|}
\hline \multirow[b]{3}{*}{ Edad (días) } & \multicolumn{9}{|c|}{ Categoría de peso al nacimiento } & \multirow[b]{3}{*}{$P$} \\
\hline & \multicolumn{3}{|c|}{ Bajo } & \multicolumn{3}{|c|}{ Medio } & \multicolumn{3}{|c|}{ Alto } & \\
\hline & $\mathrm{n}$ & $\mathrm{pp}$ & $\mathrm{DE}$ & $\mathrm{n}$ & $\mathrm{pp}$ & $\mathrm{DE}$ & $\mathrm{n}$ & $\mathrm{pp}$ & $\mathrm{DE}$ & \\
\hline 70 & 50 & $23.96^{\mathrm{a}}$ & 5.20 & 279 & $28.66^{b}$ & 4.07 & 52 & $30.76 c$ & 4.20 & 0.001 \\
\hline 85 & 52 & $28.66^{a}$ & 6.27 & 275 & $38.97^{b}$ & 4.59 & 49 & $40.61^{b}$ & 5.17 & 0.001 \\
\hline 100 & 49 & $30.76^{a}$ & 7.29 & 263 & $49.00^{b}$ & 5.63 & 47 & $52.03^{b}$ & 6.24 & 0.001 \\
\hline 115 & 50 & $53.83^{a}$ & 7.33 & 265 & $60.32^{\mathrm{a}}$ & 6.6 & 47 & $65.31^{b}$ & 8.31 & 0.001 \\
\hline 130 & 50 & $62.12^{\mathrm{a}}$ & 6.94 & 262 & $71.69 \mathrm{~b}$ & 7.99 & 48 & $75.98^{b}$ & 7.57 & 0.001 \\
\hline 145 & 29 & $76.64^{a}$ & 10.03 & 160 & $83.17^{b}$ & 8.56 & 36 & $85.97^{b}$ & 8.72 & 0.004 \\
\hline 160 & 46 & $86.66^{a}$ & 11.18 & 227 & $95.7^{\mathrm{b}}$ & 9.15 & 46 & $101.11^{b}$ & 12.17 & 0.001 \\
\hline
\end{tabular}


3. Oksbjerg N, Nissen PM, Therkildsen M, Moller HS, Larsen LB, Andersen M, Young JF. Meat science and muscle biology symposium: in utero nutrition related to fetal development, postnatal performance, and meat quality of pork. J Anim Sci 2013; 91:1443-1453.

4. Panzardi A, Bernardi ML, Mellagi AP, Bierhals T, Bortolozzo FP, Wentz I. Newborn piglet traits associated with survival and growth performance until weaning. Prev Vet Med 2013;110(2):206-213.

5. Quiniou N, Dagorn J, Gaudré D. Variation of piglets' birth weight and consequences on subsequent performance. Livest Prod Sci 2002; 78(1):63-70.

6. Wientjes JGM, Soede NM, Van der Peet-Schwering CMC, Van der Brand $\mathrm{H}$, Kemp B. Piglet uniformity and mortality in large organic litters: Effects of parity and pre-mating diet composition. Livest Sci 2012; 144:218-229.

7. Lawlor PG, Linch PB, O'connell MK, Mcnamara L, Reid P, Stickland NC. The influence of over feeding sows during gestation on reproductive performance and pig growth to slaughter. Arch Tierz Dummerstorf 2007; 50:82-91.

8. Mota RD, Martínez BJ, Villanueva GD, Roldan SP, Trujillo OMA Orozco GH, Bonilla JH, et al. Animal welfare in the newborn piglet: a review. Vet Med 2012; 7:338-349.

9. Marques $\mathrm{CMJ}$. Probabilidad y estadistica para ciencias químico biológicas. McGraw-Hill; 1991.

10. SAS. J mp (statistical discovery software), ver. 4.0.2 (academic); Cary NC, USA:SAS Inst.Inc. 2002.
11. Berard J, Kreuzer M, Bee G. In large litters birth weight and gender is decisive for growth performance but less for carcass and pork quality traits. Meat Sci 2010;86(3):845-851.

12. Handel SE, Stickland NC. Catch-up growth in pigs: a relationship with muscle cellularity. Anim Prod 2010;47(2):291-295.

13. Gondret F, Lefaucheur L, J uin H, Louveau I, Lebret B. Low birth weight is associated with enlarged muscle fiber area and impaired meat tenderness of the lingissimus muscle in pigs. J Anim Sci 2006; 84:93-103.

14. Beaulieu AD, Aalhus J L, Williams NH, Patience JF. Impact of piglet birth weight, birth order, and litter size on subsequent growth performance, carcass quality, muscle composition, and eating quality of pork. J Anim Sci 2010;88:2767-2778.

15. Kammersgaard TS, Pedersen LJ, Jorgensen E. Hypothermia in neonatal piglets: interactions and causes of individual differences. J Anim Sci 2011;89:2073-2085.

16. Vasdal G, Østensen I, Melišová M, Bozděchová B, Illmann G, Andersen IL. Management routines at the time of farrowing effects on teat success and postnatal piglet mortality from loose housed sows. Livest Sci 2011;136:225-231.

17. Pedersen ML. Nursing-suckilng behaviour, effects of farrowing enviroment on duration of milk letdown and piglet weight gain [Masther thesis]. Denmark, Copenhagen; University of Copenhagen; 2009.

18. Rehfeldt C, Tuchscherer A, Hartung M, Kuhn G. A second look at the influence of birth weight on carcass and meat quality in pigs. Meat Sci 2008; 78:170-175. 